\title{
Whole-Genome Sequencing for Outbreak Investigations of Methicillin-Resistant Staphylococcus aureus in the Neonatal Intensive Care Unit: Time for Routine Practice?
}

\author{
Taj Azarian, MPH, PhD; ${ }^{1,2}$ Robert L. Cook, MD; ${ }^{1,2}$ Judith A. Johnson, PhD; ${ }^{3,2}$ Nilmarie Guzman, MD; ${ }^{4}$ \\ Yvette S. McCarter, PhD; $;^{5}$ Noel Gomez; ${ }^{6}$ Mobeen H. Rathore, MD; ${ }^{7,8}$ J. Glenn Morris Jr., MD; ${ }^{2,4}$ Marco Salemi, $\mathrm{PhD}^{2,3}$
}

BACKGROUND. Infants in the neonatal intensive care unit (NICU) are at increased risk for methicillin-resistant Staphylococcus aureus (MRSA) acquisition. Outbreaks may be difficult to identify due in part to limitations in current molecular genotyping available in clinical practice. Comparison of genome-wide single nucleotide polymorphisms (SNPs) may identify epidemiologically distinct isolates among a population sample that appears homogenous when evaluated using conventional typing methods.

ов естіле. To investigate a putative MRSA outbreak in a NICU utilizing whole-genome sequencing and phylogenetic analysis to identify recent transmission events.

DESIGN. Clinical and surveillance specimens collected during clinical care and outbreak investigation.

Patients. A total of 17 neonates hospitalized in a 43-bed level III NICU in northeastern Florida from December 2010 to October 2011 were included in this study.

METHODs. We assessed epidemiological data in conjunction with 4 typing methods: antibiograms, PFGE, spa types, and phylogenetic analysis of genome-wide SNPs.

RESUlts. Among the 17 type USA300 isolates, 4 different spa types were identified using pulsed-field gel electrophoresis. Phylogenetic analysis identified 5 infants as belonging to 2 clusters of epidemiologically linked cases and excluded 10 unlinked cases from putative transmission events. The availability of these results during the initial investigation would have improved infection control interventions.

CONCLUSION. Whole-genome sequencing and phylogenetic analysis are invaluable tools for epidemic investigation; they identify transmission events and exclude cases mistakenly implicated by traditional typing methods. When routinely applied to surveillance and investigation in the clinical setting, this approach may provide actionable intelligence for measured, appropriate, and effective interventions.

Infect. Control Hosp. Epidemiol. 2015;36(7):777-785

Methicillin-resistant Staphylococcus aureus (MRSA) is a leading cause of healthcare-associated infections (HAIs), significantly contributing to morbidity and mortality of hospitalized patients. Infants in the neonatal intensive care unit (NICU) are at increased risk for infection and colonization with MRSA, often resulting in poor outcomes and long-term sequelae. ${ }^{1}$ MRSA in the NICU may be acquired from colonized parents, healthcare workers (HCWs), and other neonates. ${ }^{2,3}$ The CDC estimates that $\sim 50 \%$ of MRSA infections for patients 3-89 days old are hospital-onset cases. ${ }^{4}$ Community reservoirs have been implicated in the introduction of MRSA into NICUs by increasing colonization prevalence among patients and visitors. ${ }^{5}$ However, identifying reservoirs and tracking the source of implicated strains has proven difficult, resulting in the persistence of transmission despite aggressive control measures. ${ }^{6-8}$ Limitations in genotyping techniques available in clinical practice may hinder the investigation of MRSA outbreaks in healthcare settings. ${ }^{8}$ Genotyping is an indispensable component of epidemic detection and investigation because it discriminates among genetically similar strains for the

Affiliations: 1. College of Public Health and Health Professions and College of Medicine, Department of Epidemiology, University of Florida, Gainesville, Florida; 2. Emerging Pathogens Institute, University of Florida, Gainesville, Florida; 3. Department of Pathology, Immunology and Laboratory Medicine, University of Florida, Gainesville, Florida; 4. Department of Internal Medicine Division of Infectious Diseases, University of Florida, Jacksonville, Florida; 5. Department of Pathology and Laboratory Medicine, University of Florida, Jacksonville, Florida; 6. University of Florida Health Jacksonville, Jacksonville, Florida; 7. University of Florida, Center for HIV/AIDS Research, Education and Service (UF CARES) Gainesville, Florida; 8. Infectious Diseases and Immunology, and Infection Control and Prevention, Wolfson Children's Hospital, Jacksonville, Florida.

Received November 26, 2014; accepted March 1, 2015; electronically published May 8, 2015

(c) 2015 by The Society for Healthcare Epidemiology of America. All rights reserved. 0899-823X/2015/3607-0004. DOI: 10.1017/ice.2015.73 
identification of epidemiologically important isolates. Pulsedfield gel electrophoresis (PFGE), spa typing, antibiograms, and multilocus sequence typing (MLST) are commonly employed to investigate MRSA transmission. However, these methods may not be optimal, as the unit of categorization (eg, PFGE type, spa type, MLST profile) can encompass broad genetic and epidemiological diversity, ${ }^{6,8}$ making it difficult to differentiate sporadic from epidemic cases, ${ }^{9-14}$ particularly when a prevalent strain type is commonly identified.

MRSA PFGE-typed USA300 is an important pathogen in community and healthcare settings. In the United States, these strains were historically associated with community-associated (CA) infections acquired outside of hospitals. However, in many healthcare facilities, including those in our study area, CA-MRSA strains are displacing healthcare-associated (HA) strains as a cause of HAIs. The increasing prevalence of USA300 emphasizes the need for advanced typing methods in clinical practice. Recently, phylogenetic analysis of wholegenome sequencing (WGS) data has provided the resolution to discriminate between closely related isolates of bacterial pathogens through comparison of single nucleotide polymorphisms (SNPs). ${ }^{15,16}$ As a result, epidemiologically important isolates may be identified among a sample that appears homogenous when analyzed using conventional genotyping methods. WGS technology is often not readily available to investigators of outbreaks in the healthcare setting. ${ }^{15,17-19}$ Epidemiological linkages between patients may then be spuriously attributed and transmission sources obscured, leading to ineffective interventions and uninterrupted transmission.

We sought to determine whether phylogenetic analysis of SNPs would facilitate identification of the source of MRSA transmission amid a putative NICU outbreak, compared to the initial investigation that utilized traditional genotyping. We considered multiple typing methods including maximum likelihood (ML) and Bayesian phylogenetic analysis of SNP data. Epidemiological and phylogenetic data were covisualized to illustrate the temporal and genetic relationships among cases, allowing for assessment of patient-to-patient transmission events. We demonstrate how this approach would have enhanced the investigation, ruling out several sporadic cases of MRSA and potentially augmenting infection control interventions.

\section{MATERIALS AND METHODS}

\section{Outbreak Investigation}

The 43-bed, level 3 NICU in Hospital A provides medical care for premature and critically ill newborns, serving as a regional referral center for northeastern Florida and southern Georgia. In December 2010, surveillance of clinical specimens collected during routine clinical care identified a temporal cluster of 4 MRSA infections among NICU patients. The county health department was notified and a joint investigation was initiated together with the hospital infection control and prevention department. In January 2011, active MRSA surveillance was initiated through collection of nares swabs from neonates on admission and weekly thereafter. Prior to this period, active surveillance was not routinely implemented. Surveillance swabs were analyzed using the GeneXpert molecular diagnostic system Xpert MRSA assay (Cepheid, Sunnyvale, CA). Positive surveillance swabs were cultured on Columbia nalidixic acid agar (Becton Dickinson, Sparks, MD). Clinical samples were obtained from infants demonstrating signs of infection as part of routine clinical evaluation as determined by the clinical team. S. aureus isolates were identified using conventional biochemical methods and MRSA isolates were confirmed using cefoxitin disk diffusion. MRSA isolates were sent to the Florida Department of Health Bureau of Laboratories for PFGE typing. Health department and hospital investigators conducted a detailed investigation involving review of medical records for pertinent epidemiological data (eg, dates of admission and discharge, laboratory results, patient demographics, bed assignments, and procedures). While the investigation was underway, a number of infection control interventions were implemented. These included limited patient visitation, discontinuation of "kangaroo care" (the process by which an adult coddles the infant through skin-to-skin contact), patient cohorting, contact precautions, and enhanced environmental cleaning. A review of infection control practices was conducted, focusing on cleaning, disinfection of equipment, and medication delivery. From December 2010 to October 2011, 34 MRSA isolates were identified among unique patients. Of these, 17 ( 7 clinical and 10 surveillance) were identified as type USA300 using PFGE, including those collected from the 4 index cases. A total of 10 varying PFGE types were observed among the 17 additional isolates. Moreover, 11 of the isolates (73\%) detected in the first 5 months of the investigation were typed as USA300. After an initial review of epidemiological and laboratory data, neonates with type USA300 identified using PFGE were suspected to be epidemiologically linked through recent transmission events (Figure 1). Non-USA300 strains were investigated on a case-by-case basis and suspected to represent sporadic "background" cases. Despite the intensive epidemiological investigation, neither the origin of the epidemic strain nor the route of transmission could be identified.

\section{Sample Collection and Epidemiologic Investigation}

To determine whether phylogenetic analysis of SNP data could elucidate putative transmission events, the 17 USA300 isolates were further evaluated in our laboratory. The first positive isolate for each patient was selected for spa typing, WGS, and phylogenetic analysis. Dates of admission and discharge, positive MRSA laboratory results, and previously negative clinical and surveillance tests were used to reconstruct the epidemic timeline and assess overlapping lengths of stay (LOS). To illustrate how increasing levels of genetic resolution could be used to investigate transmission events, we created 3 epidemic curves: (1) Laboratory collection date of positive clinical and surveillance tests and PFGE analysis performed by 
the Florida Department of Health, (2) spa-typing results of PFGE-typed USA300 isolates, and (3) WGS and phylogenetic analysis.

\section{WGS, Phylogenetic Analysis, and MLST+ Comparison}

Sample preparation, genomic DNA (gDNA) isolation, and spa typing were performed as previously described. ${ }^{14}$ Isolated gDNA were sequenced using Illumina HiSeq 2000. Filtered FASTQ files were mapped to MRSA reference genome FPR3757 and SNPs were called and filtered by quality and depth of coverage. SNP differences were compared between isolates. Neighbor joining (NJ) and maximum likelihood (ML) phylogenetic trees were inferred from SNP alignment.

We selected a cutoff of $\leq 30$ SNP differences to indicate a putative transmission event because studies have suggested differences ranging from 23 to 40 are indicative of epidemiological linkages between individuals. ${ }^{20,21}$ Furthermore, we previously identified a diversity of 138 SNP differences between unrelated spa-type t008 MRSA strains among healthcare facilities within our study area. ${ }^{14} \mathrm{~A}$ molecular clock was calibrated to assess the evolutionary timescale of MRSA spread utilizing a Bayesian framework. The age for each tip of the Bayesian phylogeny was represented by the isolate sampling date. To explore the feasibility and reproducibility of available software packages with the potential for implementation in the clinical or public health laboratory setting, we analyzed WGS data using SeqSphere+ software v2.0 (Ridom, Muenster, Germany) to produce genome-wide allelic profiles (MLST+). This software is one example of a graphical user interface that provides an alternative to complex bioinformatic pipelines and advanced phylogenetic analysis. NJ phylogenies from the SNP-based and MLST+ phylogenetic analyses were compared to determine concordance. (For detailed methods see Supplementary Material.)

\section{RES ULTS}

\section{Study Population, Risk Factors, and Investigation}

Identification of 4 positive clinical cultures within 10 days of admission (patients 1-4) alerted hospital infection control staff of a putative outbreak, and an investigation was initiated. Over the 10-month investigation, 17 patients with the suspected outbreak strain type USA300, were identified using PFGE. The 17 patients included 8 males (47\%) and 9 females (53\%). A timeline of the putative outbreak was obtained by reviewing admission dates, discharge dates, and report dates of relevant laboratory results (Figure 1). Comparison of patient LOS showed 2 discrete periods of the outbreak separated by 37 days including patients $1-11$ and $12-17$, leading investigators to determine that transmission was persisting despite interventions. All 17 neonates were born in the hospital and were directly admitted to the NICU, reducing the likelihood of MRSA introduction from a referring hospital. A total of 7 clinical isolates (from patients $1-4,8,13$, and 14) and 10 surveillance cultures (from patients 5-7, 9-12, and 15-17) were obtained from incident MRSA cases. A total of 6 infants had a MRSA-negative clinical culture, and 5 infants had a negative surveillance culture prior to a subsequent positive result (Figure 1). The median LOS was 19 days (range 1-33), and the median time to MRSA-positive culture result was 12 days

\begin{tabular}{|c|c|c|c|c|c|c|c|c|c|c|c|c|c|c|c|c|c|c|c|c|c|c|c|c|c|c|c|c|}
\hline Pt. & Spa & 10 & 20 & 30 & 40 & 50 & 60 & 70 & 80 & 90 & 100 & 110 & 120 & 130 & 180 & 190 & 200 & 210 & 220 & 230 & 240 & 250 & 260 & 270 & 280 & 290 & 320 & 330 \\
\hline 1 & to08 & & & & & & & & & & & & & & & & & & & & & & & & & & & \\
\hline 2 & toos & & & & & & & & & & & & & & & & & & & & & & & & & Negative Lab Result & & \\
\hline 3 & toos & & & c & & & & & & & & & & & & & & & & & & & & & & Length of Stay & & \\
\hline 4 & t5593 & & & c & & & & & & & & & & & & & & & & & & & & & & Positive MRSA Lab Result & & \\
\hline 5 & toos & & & & & & & & & & & & & & & & & & & & & & & & & Clinical Isolate & & \\
\hline 6 & toos & & s & & & & & & & & & & & & & & & & & & & & & & & Surveillance Isolate & & \\
\hline 7 & $\mathrm{t} 118$ & & & & & & & & & & & & & & & & & & & & & & & & & & & \\
\hline 8 & t118 & & & & & & & & & & & & & & & & & & & & & & & & & & & \\
\hline 9 & to08 & & & & & & & & & & & & & & & & & & & & & & & & & & & \\
\hline 10 & to08 & & & & & & & & & & & & & & & & & & & & & & & & & & & \\
\hline 11 & t008 & & & & & & & & & & & & & & & & & & & & & & & & & & & \\
\hline 12 & toos & & & & & & & & & & & & & & & $s$ & & & & & & & s & & & & & \\
\hline 13 & t211 & & & & & & & & & & & & & & & & & & & & & & & & & & & \\
\hline 14 & t211 & & & & & & & & & & & & & & & & & & & & & & & & & & & \\
\hline 15 & to08 & & & & & & & & & & & & & & & & & & & & & & & & & & & \\
\hline 16 & toos & & & & & & & & & & & & & & & & & & & & & & & & & & & \\
\hline 17 & toos & & & & & & & & & & & & & & & & & & & & & & & & & & & \\
\hline
\end{tabular}

FIGURE 1. Detailed timeline for putative neonatal intensive care unit (NICU) outbreak. Lengths of stay are indicated in grey for the 17 pulse-field gel electrophoresis type USA300 isolates. Spa-types are indicated next to the patient number. Positive (red) and previously negative (green) surveillance (S) and clinical (C) isolates are illustrated. A 37-day gap between the 2 discrete outbreak periods is designated with a double vertical line shaded in gray. 


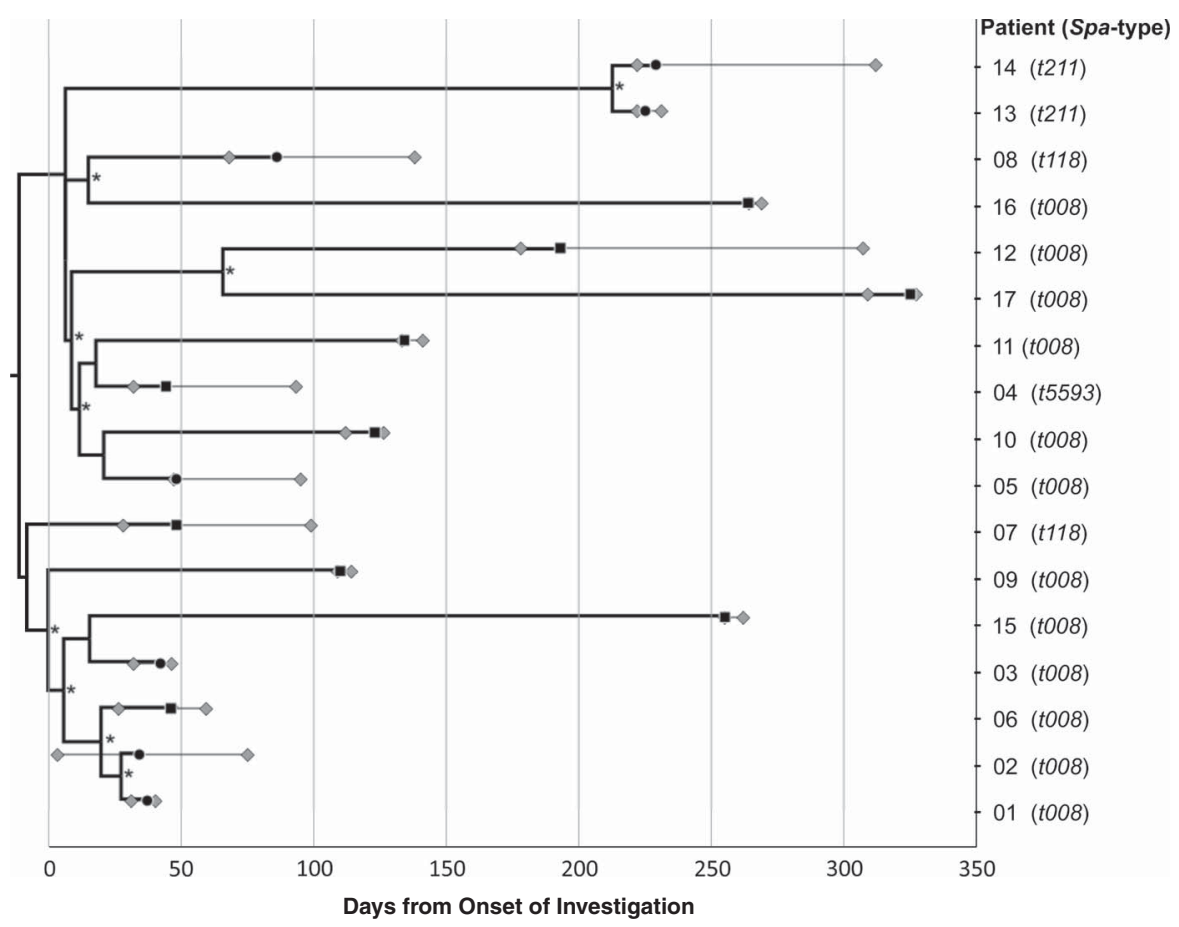

FIGURE 2. Integration of epidemiological and phylogenetic data to produce a timeline of putative neonatal intensive care unit (NICU) outbreak. The Bayesian maximum clade credibility (MCC) tree represents the phylogenetic relationship between pulsed-field gel electrophoresis (PFGE)-type USA300 isolates from 17 infants hospitalized in the NICU. The MCC phylogeny is scaled in time with tip dates, indicated by black a circle (clinical isolates) or square (surveillance isolate), corresponding to the date of incident laboratory result. The lengths of stay of each infant are represented as lines extending from the phylogeny tip dates. The first gray diamond represents the day of admission scaled by the earliest admission date among all cases. The last gray diamond represents the date of discharge. An asterisk on the branch marks subtending clades supported by posterior probability $>0.75$. The Bayesian MCC tree of 17 NICU isolates was constructed using HKY nucleotide substitution model, Bayesian Skyline demographic model, and lognormal uncorrelated (relaxed) molecular clock.

(range 1-33) from the date of admission. Colonized patients and patients with clinical cultures did not differ significantly with respect to LOS (Mann-Whitney $U=23.5 ; P>.05$ ) or days to positive result (Mann-Whitney $U=26 ; P>.05$ ).

\section{Molecular, Genomic, and Phylogenetic Results}

The primary investigation focused on 17 patients with PFGEtyped USA300 isolates (Supplementary Figure 1). Among these isolates, spa typing identified 5 isolates that were discordant from the expected $t 008$ genotype (Figure 1). SNP differences between WGS ranged from 4 to 188 (mean, 143), and those of patients 1,2 , and 6 differed by $\leq 10$ nucleotides, meeting our criteria for epidemiological linkage (Supplementary Table 2). Patients 13 and 14, who were siblings admitted on the same day, differed by only 4 nucleotides, suggesting vertical transmission through birthing or other common source; however, parents were not screened to confirm this association. The MRSA genome sequences of the 12 remaining patients differed by a minimum of 77 nucleotides. The time-scaled Bayesian maximum clade credibility phylogeny utilizes an estimated rate of molecular evolution (molecular clock) to identify divergence dates of common ancestors. In the context of our investigations, this method allowed the temporal assessment of transmission events. Isolates from patients 1, 2, and 6 were clustered within a monophyletic clade (Figure 2 and Supplementary Figure 2) and shared a recent common ancestor with those from patients $3,9,7$, and 15 . The remaining isolates clustered on a distinct clade, including the closely related sibling isolates from patients 13 and 14. The most recent common ancestor of all 17 MRSA isolates were dated just 7 weeks (95\% HPD, 1-35) prior to the admission date of the earliest patient. When genomic data were considered in the context of the epidemic curve, several transmission events were ruled out (Figure 3). Antibiotic susceptibility patterns demonstrated the ambiguity of comparing antibiograms (Supplementary Table 1). Antibiograms were consistent with CA-MRSA strains possessing resistance to oxacillin and erythromycin, with the exception of 2 isolates susceptible to erythromycin and 2 isolates intermediately resistant to levofloxacin. Phylogenetic analysis did not epidemiologically link these patients through a recent transmission event (Figure 2 and Supplementary Figure 2). SeqSphere + typed all isolates as MLST ST8, consistent with PFGE-typed USA300 genotype. Comparison of 

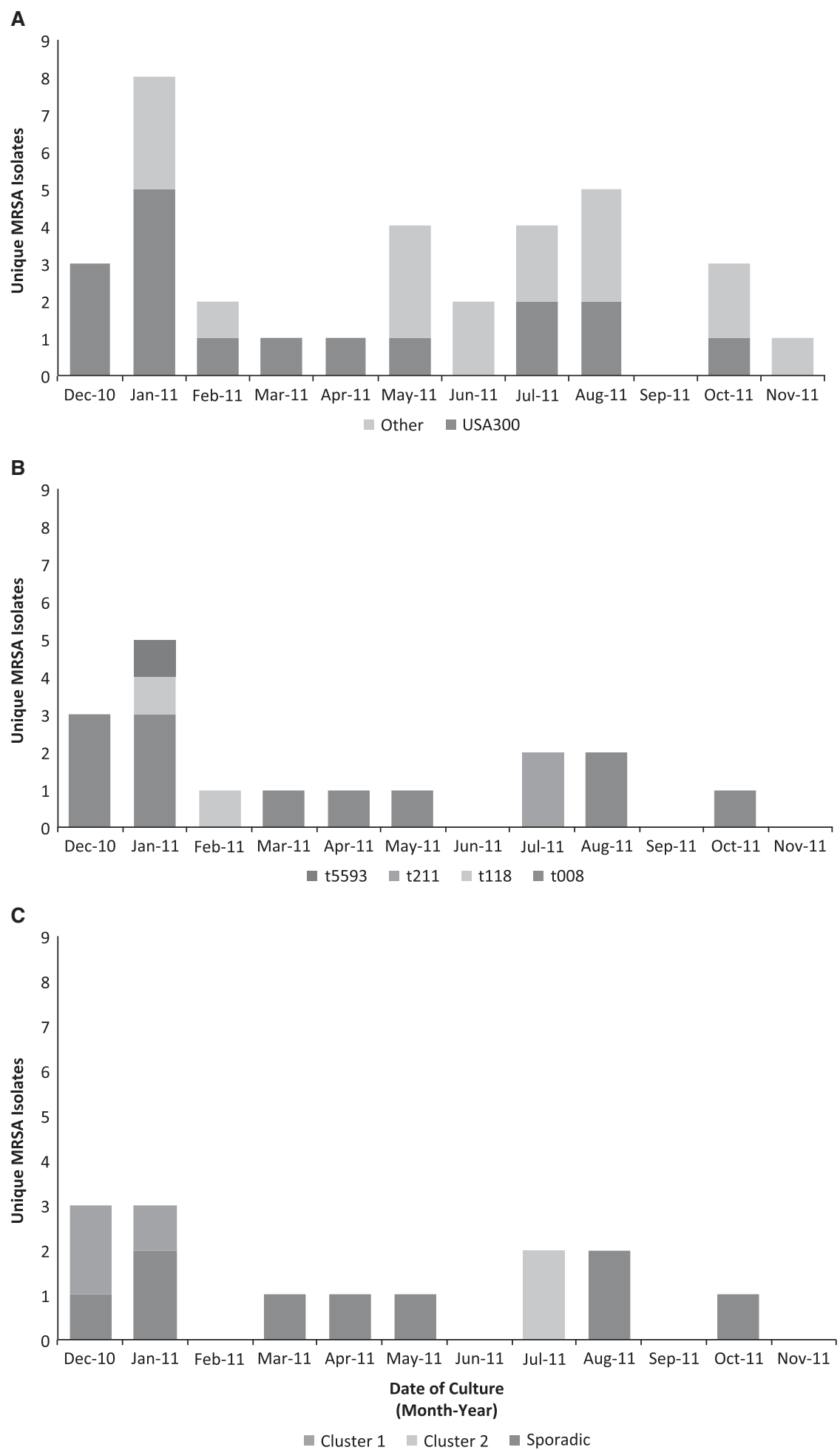

FIGURE 3. Epidemic curve of putative (neonatal intensive care unit) NICU outbreak incorporating increasing levels of resolution from molecular and whole-genome sequencing (WGS) analysis. (A) Epidemic curve of 34 cases using dates of incident clinical or surveillance MRSA-positive laboratory results. Cases identified as USA300 using pulsed-field gel electrophoresis (PFGE) ( $\mathrm{n}=17)$ during the primary outbreak investigation are indicated in blue. (B) Epidemic curve of 17 PFGE-typed USA300 cases stratified by spa-type conducted retrospectively to identify 5 non-t008 spa-types among the 17 PFGE-typed USA300 isolates. (C) Epidemic curve of 10 remaining PFGE-typed USA300 and spa-type t008 isolates further stratified by results from phylogenetic analyses. Cluster 1 (patients 1, 2, and 6) represent epidemiological linkages based on phylogenetic data (eg, SNP distances) and epidemiological assessment (eg, overlapping lengths of stay). 
A

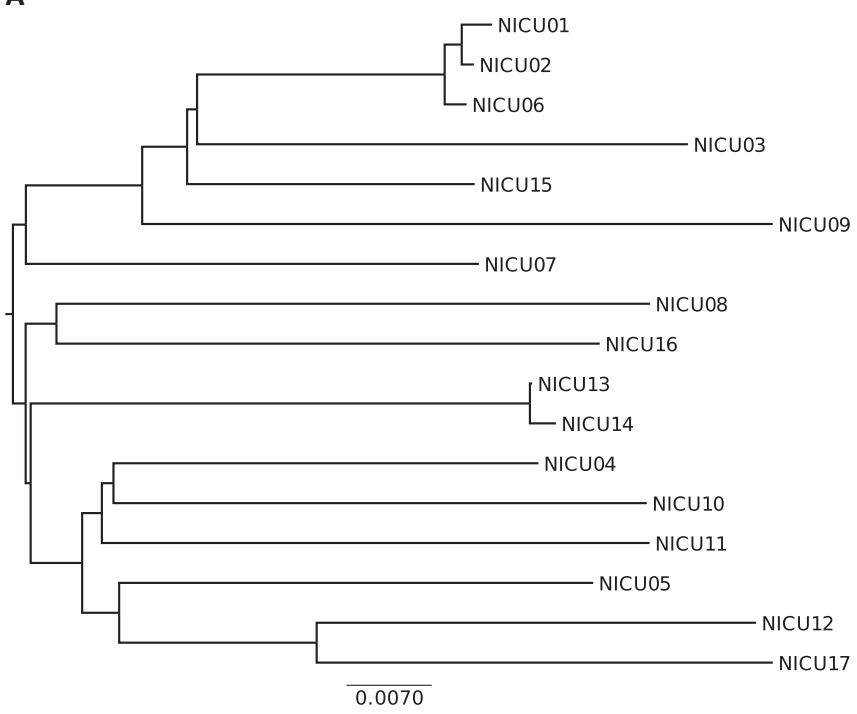

B

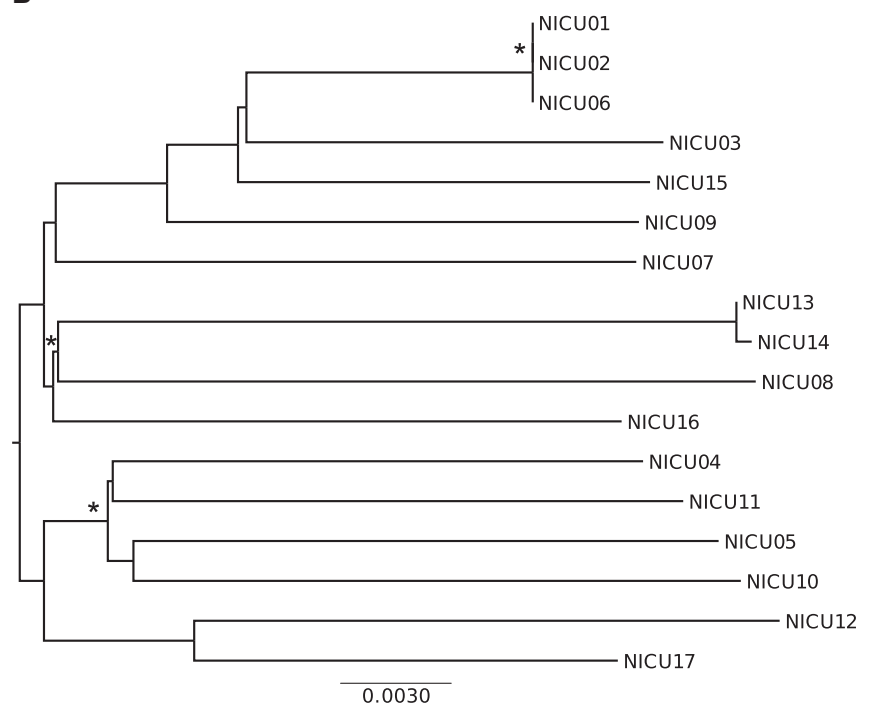

FIGURE 4. Comparison of neighbor joining (NJ) trees constructed with genome wide single-nucleotide polymorphism data (A) and MLST + allelic profiles (B). Scale bars represent genetic distances based on nucleotide substitutions per SNP site (A) and allelic distance (B). MEGA v6.0 was used to infer phylogeny A using Kimura 2-parameter nucleotide substitution model, and branching patterns were evaluated by bootstrapping (1,000 replicates). Ridom's SeqSphere software package was used to create phylogeny B using MLST+ data from assembled genomes. Differences in the clustering of isolates are indicated with an asterisk. While branch lengths and genetic distance scale vary between phylogenies, the overall topology and interpretation remain largely unchanged.

SeqSphere + MLST + phylogenies to the genome-wide SNPbased analysis produced analogous results in regard to phylogeny topology. While branch lengths varied, the topology (ie, clustering of sequence pairs) differed by 3 variations (Figure 4). Overall, topological differences between the phylogenies would not have affected the interpretation of the results in assessment of transmission events.

\section{Putative Transmission Pathways}

The integration of epidemiological and SNP data disproved several transmission events that were suspected during the initial investigation. Isolates from patients 1,2 , and 6 possessed nearly identical genomes and overlapping LOS, providing strong evidence of recent transmission and epidemiological linkage (Figure 4B). Collection dates of isolates from patients 1 and 2 were separated by just 2 days. Phylogenetic analysis suggested that the colonization in patient 6 resulted from a common exposure, as they shared a common genetic ancestor basal to patients 1 and 2. However, although patient 6 was known to have a negative blood culture on admission, a surveillance swab was not collected; therefore, it is unknown whether the patient was colonized. Twin patients 13 and 14 may have acquired the strain through vertical transmission or from a common parent, visitor, or HCW; however, transmission via a common source within the NICU cannot be ruled out. The remainder of the patients were not linked by recent transmission events. Among 17 isolates assessed by phylogenetic analysis, $12(70.5 \%)$ represented genetically unique isolates, whereas 5 patients were grouped into 2 clusters (Figure 2 and 4 ). Notably, spa typing spuriously indicated a transmission event between patients 7 and 8 , which was disproven by phylogenetic analysis.

\section{DISCUSSION}

MRSA type USA300 identified using PFGE, the prevalent cause of CA-MRSA infections, was responsible for $31.6 \%$ of healthcare-associated invasive MRSA infections in 2011. ${ }^{9,22,23}$ Models have proposed the replacement of HA-MRSA by CAMRSA strains in the healthcare setting, and recent epidemiological studies have documented this occurrence. ${ }^{24,25}$ With the increasing prevalence of USA300, advanced molecular typing methods are necessary for surveillance, outbreak detection, and epidemic investigation. These activities rely on the ability to discriminate between genetically related and unrelated isolates, identifying putative transmission events that may then be investigated further. As we have demonstrated, conventional typing methods (eg, MLST, PFGE, and spa typing) used to investigate putative outbreaks of $S$. aureus provide a macro level discriminatory resolution and are unable to resolve the epidemiological relationships among genetically similar isolates. ${ }^{26-30}$ More generally, when the spa-type or PFGE pattern of the putative outbreak strain has a high prevalence within a setting (eg, hospital, healthcare system, or community), it becomes increasingly difficult for investigators to interpret 
molecular typing results, to rule out transmission events, and to identify epidemiologically linked patients.

At the onset of the NICU investigation, a cluster of MRSA infections alerted hospital infection control staff to a suspected outbreak. The subsequent implementation of active surveillance identified several neonates colonized with prevalent CA-MRSA strains, further obscuring the relationship between epidemic and sporadic (ie, background or endemic) cases. In the absence of a clear mode of transmission, nonspecific interventions were employed, yet new cases continued to emerge. ${ }^{1,31}$ Outbreak investigation and response rely heavily on laboratory data to inform case investigation and to determine appropriate interventions. Because certain infection control interventions such as contact precautions and isolation may decrease staff interaction with patients to provide the necessary care, targeted inventions are required to stop transmission while mitigating unwanted outcomes. ${ }^{6,32,33}$

Two possible MRSA transmission scenarios may have emerged from the real-time, prospective utilization of WGS and phylogenetic analysis during the investigation. Each scenario would have then dictated distinct infection control interventions. First, close phylogenetic relationship among most or all isolates would have indicated recent transmission events, focusing the investigation on transmission pathways within the NICU. This finding may have warranted review of HCW patient assignments, medical procedures, devices, medication administration, and bassinet locations. ${ }^{1}$ General interventions may have also involved screening of HCWs, enhanced environmental cleaning, and hand hygiene compliance surveillance. Identification of colonized neonates through active surveillance or universal decolonization, as has been recently proposed, may have also been implemented. ${ }^{6}$ Secondly, and consistent with our findings, distant phylogenic relationships among isolates ruled out recent transmission events for a majority of cases. This finding indicated multiple introductions of diverse MRSA strains into the NICU, focusing interventions on colonization prevalence among parents, visitors, and newly admitted patients. Interventions may then have involved parent/caregiver screening and decolonization and a review of infection control protocols for patient visitation and patient-parent interaction (eg, kangaroo care). ${ }^{1,34}$ Ultimately, the increased cost, diminished effectiveness, and potential adverse outcomes of nontargeted interventions may have been averted using phylogenetic analysis results to guide the investigation.

Phylogenetic analysis is continually improving our knowledge of MRSA epidemiology in the NICU. ${ }^{35-37}$ Utilizing WGS data, Harris et $\mathrm{al}^{37}$ successfully tracked MRSA between the community and hospital, documenting transmission between infants and parents in the postnatal ward and eventually identifying a colonized HCW as the source. ${ }^{37}$ Both Koser et $\mathrm{al}^{35}$ and Nubel et $\mathrm{al}^{36}$ identified several epidemiological linkages among neonates based on WGS data. In contrast, we found that a large proportion of the cases were unrelated. Our findings are more consistent with those of Price et $\mathrm{al}^{20}$ who demonstrated that only $18.9 \%$ of S. aureus transmission events in the ICU were linked to other colonized patients, concluding that patient-to-patient transmission was rare in that setting. The diversity of isolates in our study may reflect a larger epidemic within the hospital or community, which would require more extensive sampling of environmental fomites, HCWs, parents in the peri- and postnatal periods, and visitors. Prospective studies should incorporate such sampling strategies to identify potential reservoirs serving as sources of MRSA introduction into the NICU. As with similar studies, MRSA surveillance in the NICU may not capture the true prevalence of colonization because neonates may be discharged before surveillance cultures are collected. Additionally, some patients may become colonized or develop infection after discharge and would be missed in the absence of follow-up or hospital readmission. As a result, some transmission events between patients may have been missed.

In conclusion, phylogenetic analysis is rapidly emerging as the next stage in the evolution in molecular (now termed genomic) epidemiology. ${ }^{15}$ The application of WGS additionally extends to the determination of genotypic antibiotic resistance and virulence. ${ }^{38,39}$ WGS may provide improved turnaround time for diagnostic purposes with the added benefit of enhanced surveillance, outbreak detection, and investigation. With the availability of benchtop sequencers (eg, Illumina's MiSeq), real-time analysis delivering actionable results is increasingly feasible. ${ }^{16,19}$ These technologies are further facilitated by the development of streamline software packages, such as Ridom's SeqSphere, that reduce computational demands and simplify analysis. While this technology may not be readily available in all clinical laboratories, the transition will likely follow that of previous technology such as automated PCR (eg, GeneXpert). Meanwhile, public health reference laboratories in the Centers for Disease Control and Prevention's laboratory response network already provide the infrastructure for widespread implementation of WGS. Last, as with all laboratory methods, WGS alone cannot replace the need for surveillance and epidemiological investigation. ${ }^{40}$ WGS should be employed in concert with traditional epidemic investigation techniques.

\section{ACKNOWLEDGMENTS}

Financial support: This work was supported in part by the NIH/NCATS Clinical and Translational Science Award to the University of Florida (no. UL1 TR000064).

Potential conflicts of interest: All authors report no conflicts of interest relevant to this article.

Address all correspondence to Taj Azarian, PO Box 103633, Gainesville, FL 32610 (taj.azarian@epi.ufl.edu) and/or Marco Salemi PO Box 103633, Gainesville, FL 32610 (salemi@pathology.ufl.edu).

\section{SUPPLEMENTARY MATERIAL}

To view supplementary material for this article, please visit http://dx.doi.org/ 10.1017/ice.2015.73 


\section{REFERENCES}

1. Gerber SI, Jones RC, Scott MV, et al. Management of outbreaks of methicillin-resistant Staphylococcus aureus infection in the neonatal intensive care unit: a consensus statement. Infect Control Hosp Epidemiol 2006;27:139-145.

2. Saiman L, Cronquist A, Wu F. An outbreak of methicillinresistant Staphylococcus aureus in a neonatal intensive care unit. Infect Control Hosp Epidemiol 2003;24:317-321.

3. Geva A, Wright SB, Baldini LM, Smallcomb JA, Safran C, Gray JE. Spread of methicillin-resistant Staphylococcus aureus in a large tertiary NICU: network analysis. Pediatrics 2011;128:e1173-e1180.

4. Iwamoto $\mathrm{M}, \mathrm{Mu} \mathrm{Y}$, Lynfield $\mathrm{R}$, et al. Trends in invasive methicillin-resistant Staphylococcus aureus infections. Pediatrics 2013;132:e817-e824.

5. Zervou FN, Zacharioudakis IM, Ziakas PD, Mylonakis E. MRSA colonization and risk of infection in the neonatal and pediatric ICU: a meta-analysis. Pediatrics 2014;133:e1015-e1023.

6. Popoola VO, Budd A, Wittig SM, et al. Methicillin-resistant Staphylococcus aureus transmission and infections in a neonatal intensive care unit despite active surveillance cultures and decolonization: challenges for infection prevention. Infect Control Hosp Epidemiol 2014;35:412-418.

7. Popoola VO, Carroll KC, Ross T, Reich NG, Perl TM, Milstone AM. Impact of colonization pressure and strain type on methicillin-resistant Staphylococcus aureus transmission in children. Clin Infect Dis 2013;57:1458-1460.

8. Mangini E, Srinivasan P, Burns J, et al. Unrelated strain methicillin-resistant Staphylococcus aureus colonization of health care workers in a neonatal intensive care unit: Findings of an outbreak investigation. Am J Infect Control 2013;41. doi:10.1016/ j.ajic.2013.05.017.

9. Otter JA, French GL. Community-associated methicillin-resistant Staphylococcus aureus strains as a cause of healthcare-associated infection. J Hosp Infect 2011;79:189-193.

10. Klevens RM, Edwards JR, Tenover FC, McDonald LC, Horan T, Gaynes R. Changes in the epidemiology of methicillin-resistant Staphylococcus aureus in intensive care units in US hospitals, 1992-2003. Clin Infect Dis 2006;42:389-391.

11. Otter JA, French GL. Molecular epidemiology of communityassociated meticillin-resistant Staphylococcus aureus in Europe. Lancet Infect Dis 2010;10:227-239.

12. Fridkin S, Hageman J, Morrison M. Methicillin-resistant Staphylococcus aureus disease in three communities. New Engl J Med J 2005;352:1436-1444.

13. Maraqa NF, Aigbivbalu L, Masnita-Iusan C, et al. Prevalence of and risk factors for methicillin-resistant Staphylococcus aureus colonization and infection among infants at a level III neonatal intensive care unit. Am J Infect Control 2011;39:35-41.

14. Prosperi M, Veras N, Azarian T, et al. Molecular epidemiology of community-associated methicillin-resistant Staphylococcus aureus in the genomic era: a cross-sectional study. Sci Rep 2013;3:1902.

15. Gardy J. Investigation of disease outbreaks with genome sequencing. Lancet Infect Dis 2012;3099:1-2.

16. Price J, Gordon NC, Crook D, Llewelyn M, Paul J. The usefulness of whole genome sequencing in the management of Staphylococcus aureus infections. Clin Microbiol Infect 2013;19:784-789.

17. Diep BA. Use of whole-genome sequencing for outbreak investigations. Lancet Infect Dis 2012;3099:10-11.
18. Le VTM, Diep BA. Selected insights from application of wholegenome sequencing for outbreak investigations. Curr Opin Crit Care 2013. doi:10.1097/MCC.0b013e3283636b8c.

19. Eyre DW, Golubchik T, Gordon NC, et al. A pilot study of rapid benchtop sequencing of Staphylococcus aureus and Clostridium difficile for outbreak detection and surveillance. BMJ Open 2012;2. doi:10.1136/bmjopen-2012-001124.

20. Price JR, Golubchik T, Cole K, et al. Whole-genome sequencing shows that patient-to-patient transmission rarely accounts for acquisition of Staphylococcus aureus in an intensive care unit. Clin Infect Dis 2014;58:609-618.

21. Uhlemann A-C, Dordel J, Knox JR, et al. Molecular tracing of the emergence, diversification, and transmission of $S$. aureus sequence type 8 in a New York community. Proc Natl Acad Sci 2014. doi:10.1073/pnas.1401006111.

22. Como-Sabetti KJ, Harriman KH, Fridkin SK, Jawahir SL, Lynfield R. Risk factors for community-associated Staphylococcus aureus infections: results from parallel studies including methicillinresistant and methicillin-sensitive $S$. aureus compared to uninfected controls. Epidemiol Infect 2011;139:419-429.

23. Active bacterial core surveillance report, emerging infections program network, methicillin-resistant Staphylococcus aureus, 2011. Centers for Disease Control and Prevention website. http://www.cdc.gov/abcs/reports-findings/survreports/mrsa11.pdf. Published 2012. Accessed March 15, 2015.

24. David MZ, Cadilla A, Boyle-Vavra S, Daum RS. Replacement of HA-MRSA by CA-MRSA Infections at an Academic Medical Center in the Midwestern United States, 2004-5 to 2008. de Lencastre H, ed. PLoS One 2014;9:e92760.

25. D’Agata EMC, Webb GF, Horn MA, Moellering RC Jr., Ruan S. Modeling the invasion of community-acquired methicillinresistant Staphylococcus aureus into hospitals. Clin Infect Dis 2009;48:274-284.

26. Vivoni AM, Moreira BM. Application of molecular techniques in the study of Staphylococcus aureus clonal evolution-a review. Mem Inst Oswaldo Cruz 2005;100:693-698.

27. Palavecino E. Clinical, epidemiological, and laboratory aspects of methicillin-resistant Staphylococcus aureus (MRSA) infections. Methods Mol Biol 2007;391:1-19.

28. David MZ, Taylor A, Lynfield R, et al. Comparing pulsed-field gel electrophoresis with multilocus sequence typing, spa typing, staphylococcal cassette chromosome mec (SCCmec) typing, and PCR for panton-valentine leukocidin, arcA, and opp3 in methicillin-resistant Staphylococcus aureus isolates. J Clin Microbiol 2013;51:814-819.

29. Okuma K, Iwakawa K, Turnidge JD, et al. Dissemination of new methicillin-resistant Staphylococcus aureus clones in the community. J Clin Microbiol 2002;40:4289-4294.

30. Carey AJ, Della-Latta P, Huard R, et al. Changes in the molecular epidemiological characteristics of methicillin-resistant Staphylococcus aureus in a neonatal intensive care unit. Infect Control Hosp Epidemiol 2010;31:613-619.

31. Siegel JD, Rhinehart E, Jackson M, Brennan PJ, Bell M. Management of mult-drug resistant organisms in healthcare settings, 2006. Centers for Disease Control and Prevention website. http://www.cdc.gov/hicpac/pdf/MDRO/MDROGuideline2006. pdf. Published 2006. Accessed March 15, 2015.

32. Morgan DJ, Diekema DJ, Sepkowitz K, Perencevich EN. Adverse outcomes associated with Contact Precautions: a review of the literature. Am J Infect Control 2009;37:85-93. 
33. Deeny SR, Cooper BS, Cookson B, Hopkins S, Robotham JV. Targeted versus universal screening and decolonization to reduce healthcare-associated meticillin-resistant Staphylococcus aureus infection. J Hosp Infect 2013;85:44-33.

34. Heinrich N, Mueller A, Bartmann P, Simon A, Bierbaum G, Engelhart S. Successful management of an MRSA outbreak in a neonatal intensive care unit. Eur J Clin Microbiol Infect Dis 2011;30:909-913.

35. Köser CU, Holden MTG, Ellington MJ, et al. Rapid wholegenome sequencing for investigation of a neonatal MRSA outbreak. N Engl J Med 2012;366:2267-2275.

36. Nübel U, Nachtnebel M, Falkenhorst G, et al. MRSA transmission on a neonatal intensive care unit: epidemiological and genome-based phylogenetic analyses. de Lencastre H, ed. PLoS One 2013;8:e54898.
37. Harris SR, Cartwright EJ, Török ME, et al. Whole-genome sequencing for analysis of an outbreak of meticillin-resistant Staphylococcus aureus: a descriptive study. Lancet Infect Dis 2012;3099:1-7.

38. Gordon NC, Price JR, Cole K, et al. Prediction of Staphylococcus aureus antimicrobial resistance from whole genome sequencing. J Clin Microbiol 2014;52:1182-1191.

39. Reuter S, Ellington MJ, Cartwright EJP, et al. Rapid bacterial whole-genome sequencing to enhance diagnostic and public health microbiology. JAMA Intern Med 2013;173:1397-1404.

40. Senn L, Zanetti G, Bally F, et al. Investigation of classical epidemiological links between patients harbouring identical, non-predominant meticillin-resistant Staphylococcus aureus genotypes and lessons for epidemiological tracking. J Hosp Infect 2011;79:202-205. 